\title{
Lattice Design of Beijing Light Source
}

\author{
N. Huang, L. Jin, D. Wang, L. Wang, A. Xiao, G. Xu \\ Institute of High Energy Physics, Chinese Academy of Sciences \\ P. O. Box 918-9, Beijing 100039, China
}

\begin{abstract}
In this paper the preliminary design of magnet lattice of Beijing Light Source(BLS) is described. Beijing Light Source is a new generation light source with the energy of $2.2 \mathrm{GeV}$ $2.5 \mathrm{GeV}$. By choosing the FBA(five bend achromat) and TBA (triplet bend achromat) structure very low nature horizontal emittance is reached with using superconducting dipole magnets. Having made some harmonic correction the acceptable dynamic aperture is obtained though further improvement is being done to enlarge horizontal aperture.
\end{abstract}

\section{DESIGN GOAL OF BLS}

Since 1990 China has been planned to build a new generation light source within the medium energy range(1.5GeV-2.0GeV).[1][2] Recently the synchrotron radiation user community in China has been showing more and more interest to hard X-ray synchrotron radiation light. On the other hand it is difficult for China to afford a $6-8 \mathrm{GeV}$ light source which can provide $20-50 \mathrm{KeV}$ critical energy photons from normal dipoles and IDs. Inspired by the ideas from some Laboratories (PSI, LBL, Daresbury, etc.)[3] we adopted the design philosophy that integrate several superconducting magnets into lattice of storage ring to provide many hard X-ray beamlines for synchrotron radiation users. Meanwhile we try to keep the horizontal emittance very small to yield high brightness VUV and soft X-ray synchrotron radiation light from insertion devices(undulators and wigglers).

The overall design goal of Beijing Light Source is listed as follows:

* Energy: $2.2 \mathrm{GeV}-2.5 \mathrm{GeV}$

* Nature horizontal emittance: $<10 \mathrm{~nm}$, better less than

* Circumference: $<250 \mathrm{~m}$

* Number of straight sections: $\geq 8$

* Length of straight sections: two long straight, $\sim 15 \mathrm{~m}$ $>6.0 \mathrm{~m}$ for the rest

* Number of superconducting dipole: 4 at least

* Beam current: $\quad 300 \mathrm{~mA}$

The figure 1 gives a comparison of the spectrum of BLS to that of two existing synchrotron light sources in China: BSRF(dedicated synchrotron radiation mode of Beijing Electron-Positron Collider, $2.2 \mathrm{GeV})$ and $\operatorname{NSRL}(0.8 \mathrm{GeV}$ synchrotron light source)

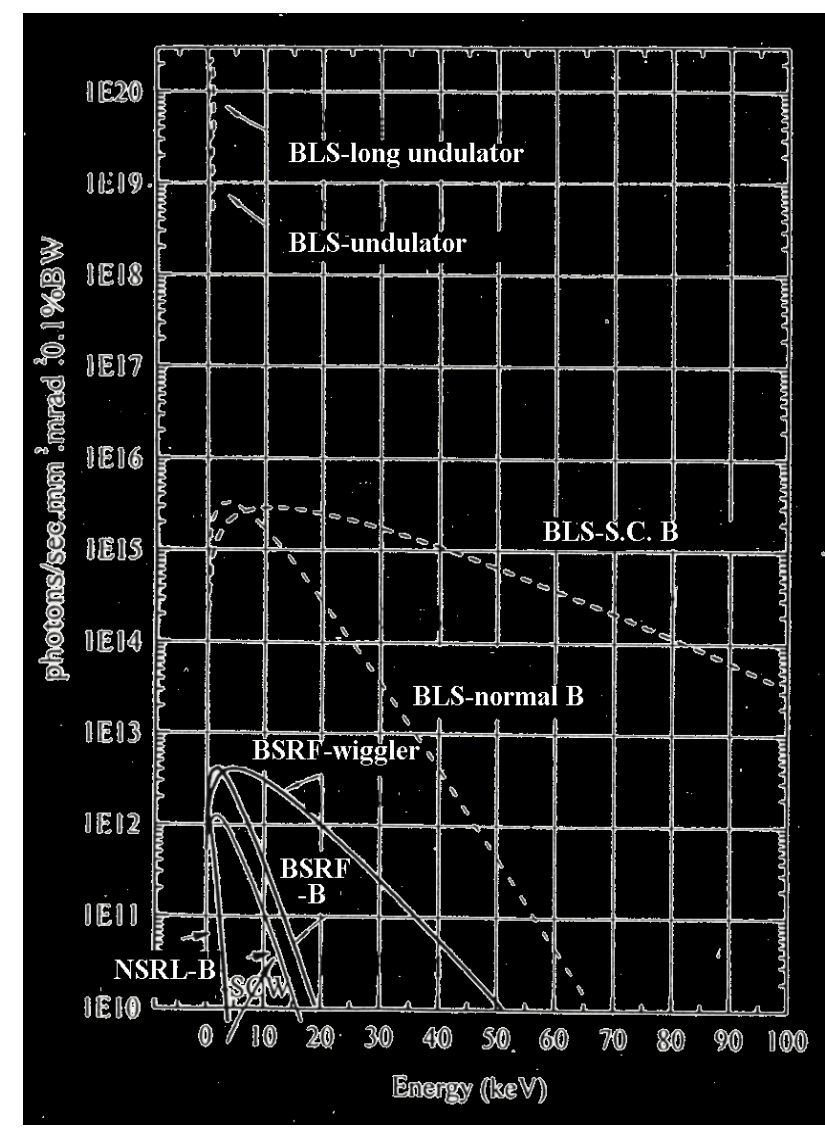

Figure 1 Comparison of the synchrotron radiation spectrum of BLS, BSRF and NSRL

\section{FBA LATTICE DESCRIPTION}

We have chosen the achromat structure with odd number of dipole magnets $(\mathrm{N}=3,5)$ in order to arrange a superconducting dipole by replacing the central normal dipole. FBA(Five Bend Achromat) structure can leads to a very low nature emittance even with the presence of superconducting bending magnets. Table 1 gives the main lattice parameters of BLS storage ring based on this kind of structure.

Each FBA cell consists of 5 bending magnets. The bending angle of outer dipoles for dispersion suppression and the central dipole which can be replaced by a superconducting dipole is chosen to be $8^{\circ}$. The bending angle of the rest 2 
dipoles is $10.5^{\circ}$. Such a distribution of bending angle is to optimize the contribution from different dipoles to emittance. The combined function dipole is adopted to save some space therefore make it possible to arrange eight FBA cells in such a rather limited circumference. The exception is that the superconducting dipoles are flat type to avoid some technological complexities. Two extra quadrupoles for each superconducting dipole are added to provide necessary focusing.

The circumference of storage ring is fixed to $240.4 \mathrm{~m}$ which is equal to that of Beijing Electron-Positron Collider therefore the design is also suitable for the case that a Taucharm Factory is built at IHEP site(build BLS in BEPC tunnel).[4][5] However we found that a little bit longer circumference $(250 \mathrm{~m}$ or $260 \mathrm{~m})$ is more comfortable for the matching and element arrangement.

Table 1 Main lattice parameters of BLS storage ring

\begin{tabular}{|l|l|}
\hline Nominal Beam Energy & $2.2 \mathrm{GeV}$ \\
Maximum Beam Energy & $2.5 \mathrm{GeV}$ \\
Circumference & $240.4 \mathrm{~m}$ \\
Nature Horizontal Emittance & $3.3 \mathrm{~nm} \cdot \mathrm{rad}$ \\
Number of Straight Section & 8 \\
Length of Long Straight & $15.0 \mathrm{~m}$ \\
Length of short straight section & $6.4 \mathrm{~m}$ \\
Number of S.C. dipole & 4 \\
Field Strength of S.C. Dipole & $4.4 \mathrm{Tesla}$ \\
Momentum Compaction & 0.00834 \\
Energy Spread & 0.00105 \\
Qx & 18.38 \\
Qy & 7.15 \\
Energy Loss per turn & $441 \mathrm{KeV}$ \\
Jx/Jy/Jz & $1.21 / 1.0 / 1.79$ \\
RMS Beam Size at ID & $0.162 / 0.036 \mathrm{~mm}$ \\
Chromaticities & $-47.2 /-19.3$ \\
Harmonic number & 400 \\
RF frequency & $498.83 \mathrm{MHz}$ \\
\hline
\end{tabular}
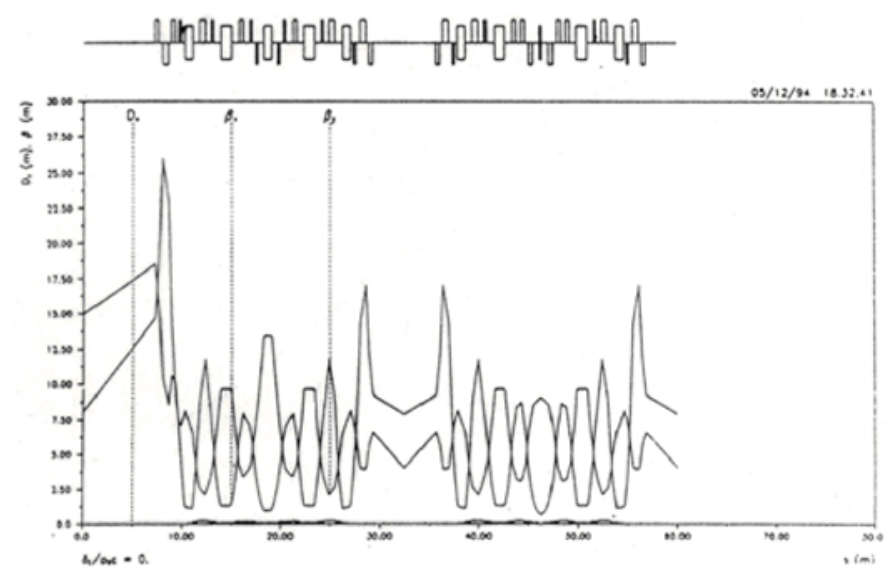

Figure 2 Lattice of $1 / 4$ ring of BLS
Table 2 The effect of s.c. dipole on nature hori. emittance

\begin{tabular}{|c|c|c|c|}
\hline Num. of SCB & $2.0 \mathrm{GeV}$ & $2.2 \mathrm{GeV}$ & $2.5 \mathrm{GeV}$ \\
\hline 0 & $1.9 \mathrm{~nm} \cdot \mathrm{rad}$ & $2.3 \mathrm{~nm} \cdot \mathrm{rad}$ & $3.0 \mathrm{~nm} \cdot \mathrm{rad}$ \\
\hline 4 & $2.7 \mathrm{~nm} \cdot \mathrm{rad}$ & $3.3 \mathrm{~nm} \cdot \mathrm{rad}$ & $3.7 \mathrm{~nm} \cdot \mathrm{rad}$ \\
\hline 8 & $3.3 \mathrm{~nm} \cdot \mathrm{rad}$ & $4.2 \mathrm{~nm} \cdot \mathrm{rad}$ & $5.2 \mathrm{~nm} \cdot \mathrm{rad}$ \\
\hline
\end{tabular}

\section{CHROMATICITY CORRECTION AND DYNAMIC APERTURE}

The correction of chromaticity with two families of sextupoles results in a rather small dynamic aperture $(\sim 7 \mathrm{~mm}$ in horizontal direction and $\sim 6 \mathrm{~mm}$ in vertical direction). Having applied two harmonic correction sextupole families located in the dispersion-free region the dynamic aperture is doubled in both directions. Further studies are being performed to enlarge the dynamicaperture by increasing the number of families of both chromaticity correction and harmonic correction sextupoles.

\section{TBA LATTICE CONFIGURATION}

As a comparison to FBA structure the TBA(Triplet Bend Achromat) configuration is done. A small nature horizontal emittance $8.5 \mathrm{~nm} \cdot \mathrm{rad}$ at $2.2 \mathrm{GeV}$ with 4 superconducting dipoles integrated in lattice is achieved by applying strong focusing strength. This configuration gives 4 more straight sections than FBA lattice though the adjustment of betafunctions in the straight section is not very flexible. By using 8 sextupole families a good dynamic aperture is obtained $(20 \mathrm{~mm}$ in horizontal and $8 \mathrm{~mm}$ in vertical). This configuration also has two $15 \mathrm{~m}$ long straight sections as FBA configuration.

\section{ACKNOWLEDGMENT}

The authors wish to thank the other members of accelerator physics group of IHEP for their useful discussions.

\section{REFERENCES}

[1] L. Chen, S. Fang, D. Wang, W. Wang, MainlandTaiwan joint whorshop on synchrotron radiation, 1993

[2] D. Ding, S. Fang, D. Xian, Internal report, 1993

[3] A. Baldereschi et al., Conceptual Design of the Swiss Synchrotron Light Source,

[4] S. Wang, this conference

[5] N. Huang et al., this conference 


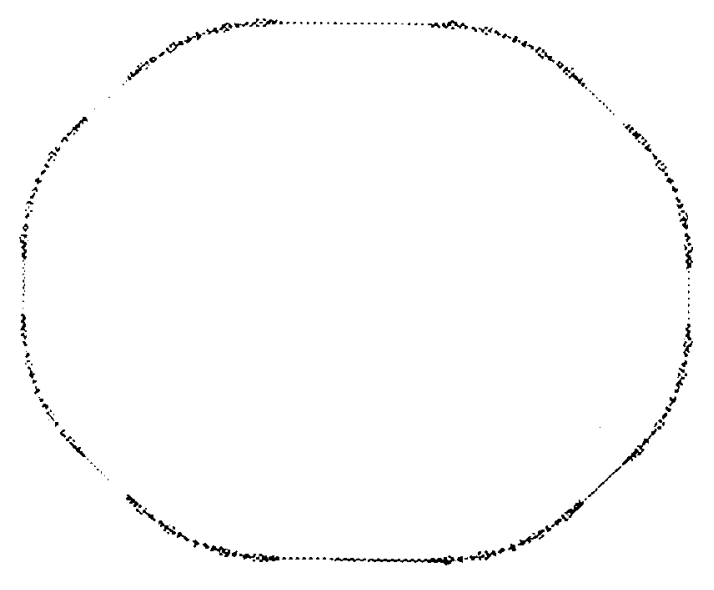

Fig. 3 Layout of the storage ring of BLS

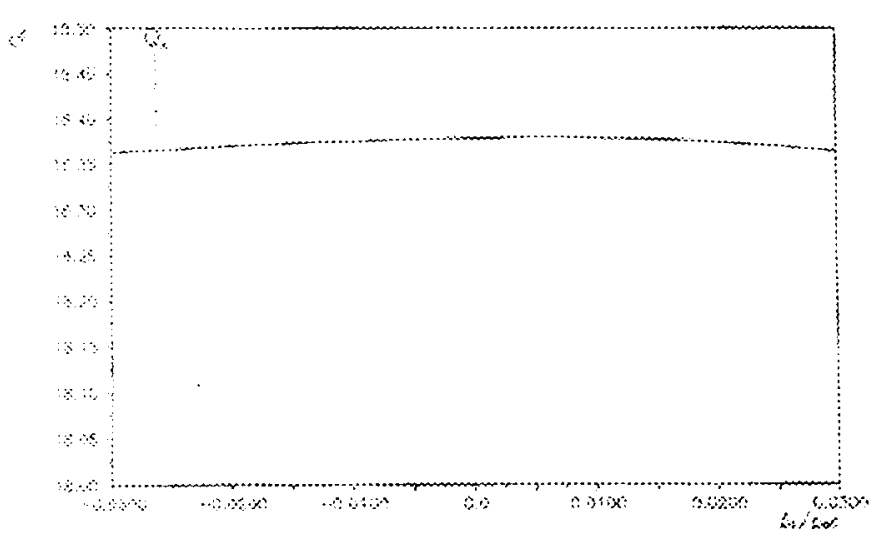

Fig. 5 Qx vs. momentum deviation

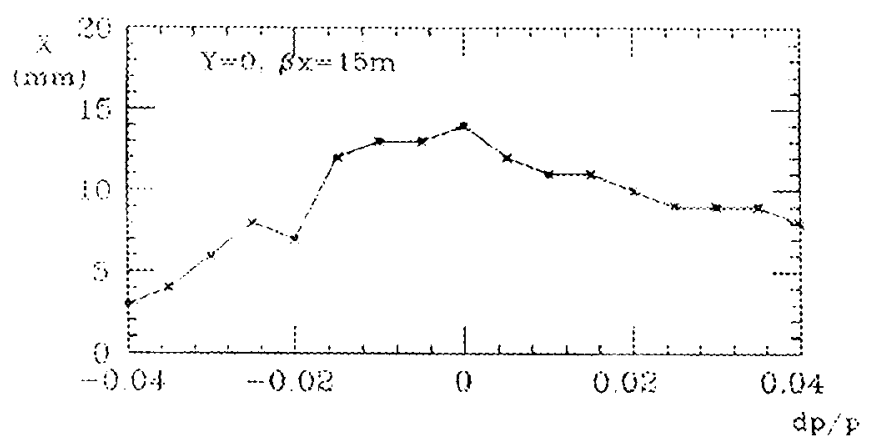

Fig. 7 Hori. dynamic aperture vs. momentum deviation

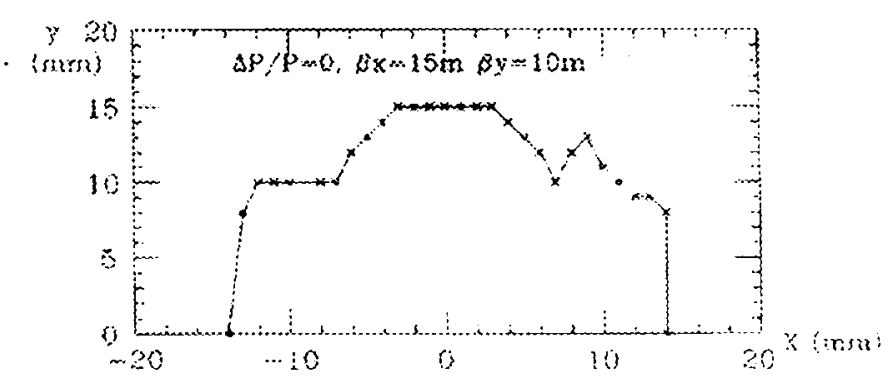

Fig. 4 Dynamic aperture of on momentum particle

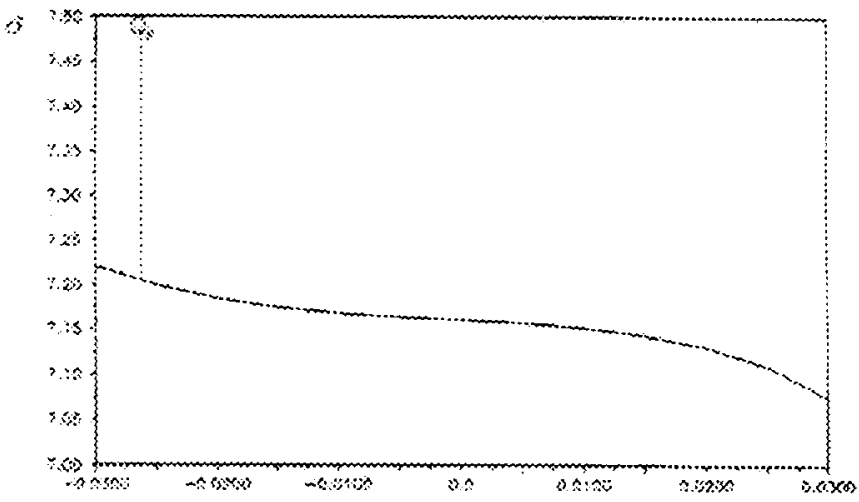

Fig. 6 Qy vs. momentum deviation

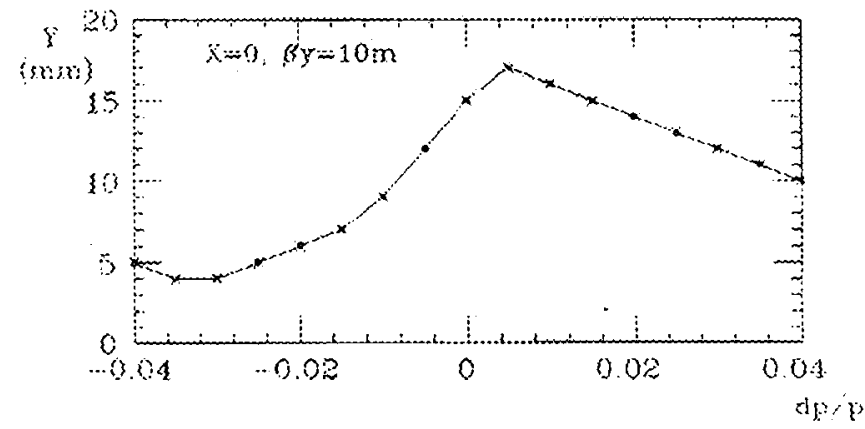

Fig. 8 Vert. dynamic aperture vs. momentum deviation 